\title{
Sobre el tiempo: primeras aproximaciones entre el pensamiento físico y el pensamiento sociológico de Norbert Elias
}

\author{
Maria J. F. GEBARA \\ Departamento de Física, Química e Matemática, Universidade Federal de São Carlos, Brasil \\ maria.gebara@ufscar.br \\ Marcos A. FLORCZAK \\ Departamento Académico de Física, Universidade Tecnológica Federal do Paraná, Brasil \\ florczak@utfpr.edu.br
}

Recibido: 10-09-2012

Aceptado: 19-12-2012

\begin{abstract}
Resumen
La concepción fundamental del tiempo en la Física es aquella construida por Galileo, al observar el aparente isocronismo de un péndulo, instrumento empleado como generador de intervalos temporales para investigar los fenómenos naturales que ocurrían en la órbita de los satélites de Júpiter. En aquel momento prevalecía una concepción divina del tiempo, en la que los objetos celestes, infalibles y sin las imperfecciones del mundo cotidiano, regían la evolución temporal de todos los fenómenos: con Galileo medimos el tiempo y a partir de él comenzamos a medir el mundo. Con Newton se produce un retorno a la concepción divina del tiempo: toda evolución es un espacio y tiempo absolutos entendidos en última instancia como sensación divina. El tiempo absoluto permea todo lo que ocurre en nuestro universo. No importa donde se encuentre el reloj ya que éste es capaz de medir los intervalos de tiempo de forma uniforme. El espacio tridimensional es euclidiano, y el tiempo totalmente independiente. Para Elias, a las tres dimensiones físicas, se le añaden otras dos construidas con base en las relaciones interdependientes entre los seres humanos: tiempo y símbolo. El tiempo es una dimensión relevante en el pensamiento de Elias, tiempo como experiencia de duración, con referencia mensurable y perspectiva de cambio que se bifurca en tiempo físico y tiempo social. El perfeccionamiento de los relojes desempeña un papel fundamental en nuestro conocimiento del mundo: en tanto instrumentos de medición, ellos mismos son productores de una dimensión del tiempo. Sin embargo no se construyeron con ese único propósito pues su precisión esta también íntimamente relacionada con las grandes navegaciones. La exactitud en la medición del tiempo ofrece mayor precisión a la hora de determinar la posición de un barco en el globo terráqueo. Con Einstein la sincronía absoluta de los relojes pierde significado: éstos medirán a partir de ahora intervalos de tiempo diferentes de acuerdo con su localización y su movimiento en el espacio. El tiempo se ha "especializado" y disponemos de un espacio como tiempo encargado de la localización de cualquier evento en nuestro universo. El propósito de este artículo es explorar las posibilidades de diálogo pertinentes en las concepciones del tiempo que maneja una ciencia natural, la Física, y las que derivan de un abordaje sociológico figuracional.
\end{abstract}

Palabras clave: tiempo; física; sociología figuracional; Norbert Elias. 


\title{
On Time: Initial Approaches between Physics and the Sociological Thought of Norbert Elias
}

\begin{abstract}
The fundamental concept of time used by Physics was built by Galileo, through the observation of the apparent pendulum isochronism and establishing the possibility of using an instrument as a generator of temporal intervals to investigate natural phenomena occurring around them. At that time, a religious conception of time was dominant: according to this, the celestial objects, infallible and without the imperfections of the common world, dictated the temporal evolution of every phenomena. Thanks to Galileo, we are able to measure time and, consequently, we started to measure the world. With Newton, we return to a divine conception of time: evolution is an absolute space and time which can be understood as a divine sensation. The absolute time permeates everything that happens in our universe; it does not matter where the clock is because it is always capable of measuring uniformly time intervals. The three-dimensional space is Euclidean, meanwhile time is completely independent. For Elias, beyond the three physical and classical dimensions, it is needed to add are two other dimensions which are built in the context of human interdependency, namely, time and symbol. Time is an relevant dimension of Elias thought, time as an experience of duration, reference, and as a measurable change; time, simultaneously, as a physical and social entity. Clocks play a key role in the way we investigate the world and as instruments of time measurement produce a new time dimensions. But they are not built just for this purpose; their accuracy is closely related to the great voyages. Accuracy in time measurement provides a more exact position of a ship's longitude on the globe. With Einstein, this absolute synchronicity of two clocks loses its meaning: from this moment, clocks measure different time intervals according to their location and spatial movement. The time is "spatialized" and space-time is now a locating system of any event in our universe. The purpose of this article is to explore the possibilities of a dialogue on concepts of time, between a natural science, Physics, and the multiple faces offered by figurational approach.
\end{abstract}

Key words: time; physics; figuracional sociology; Norbert Elias

\section{Referencia normalizada}

Gebara, M.J.F., Florczak, M.A. (2013). "Sobre el tiempo: primeras aproximaciones entre el pensamiento físico y el pensamiento sociológico de Norbert Elias”. Política y Sociedad, Vol.50 Núm. 2: $543-552$

Sumario: Introducción. 1. El concepto de tiempo en la Física. 2.El concepto de tiempo para Norbert Elias. 3.Copérnico, Einstein y Elias. 4.Conclusiones. Bibliografía 


\section{Introducción}

¿Por qué los hombres tienen necesidad de determinar el tiempo? ¿Con qué finalidad? ¿Como conducir una discusión sobre un tema que posee una enorme influencia sobre la actitud mental y el modo de vida de las personas?

Aunque las dificultades por conceptualizar y comprender el tiempo son reconocidas tanto por aquellos que se dedican a las ciencias humanas como por quienes trabajan en el campo de las ciencias exactas, unos y otros ambicionan entender por qué el tiempo tiende a dominar nuestra manera de pensar y vivir. Relojes, agendas y horarios: el tiempo parece una exigencia de la cual nadie puede escapar, pero... ¿qué es el tiempo? ¿Qué dimensión es esa?

En Sobre el Tiempo, una obra publicada en 1984, Elias presenta su tesis: el tiempo no es concebido como un hecho trascendental externo al hombre ni está relacionado con la naturaleza, como lo planteaba Newton. Tampoco es una facultad a priori, "una forma innata de experiencia, es decir un hecho no modificable por la naturaleza humana" (Elias, 1998: 8), tal y como lo comprende el pensamiento kantiano. El tiempo es, para Elias, un fenómeno socialmente construido por los propios instrumentos de medida. Al aproximarse al modelo evolucionista de Darwin, Elias explicita una tercera manera de estudiar la cuestión del tiempo en la cual el interés reside no sólo en los procesos de larga duración o en la construcción de la propia noción del tiempo sino también en el fenómeno de la evolución.

Con el propósito de explorar las posibilidades pertinentes de diálogo entre la concepción de tiempo que maneja la Física y la que emplea el enfoque figuracional, profundamente multidisciplinar, reproducimos las palabras con las que Elias abre la introducción de Sobre el Tiempo: "Cuando no me preguntan sobre el tiempo, sé lo que es” decía un anciano repleto de sabiduría. "Cuando me preguntan, no lo sé”. Entonces, ¿por qué formulamos esta pregunta? Al examinar los problemas relativos al tiempo, aprendemos acerca de los hombres y de nosotros mismos muchas cosas que antes no discerníamos con claridad” (Elias, 1998: 7).

\section{El concepto de tiempo en la Física}

El concepto de un tiempo "físico" comienza a ser delineado en la obra y en el método científico de investigación propuesto por Galileo. En su obstinado intento por estudiar el movimiento de los cuerpos, desarrolló una metodología que combinaba una sofisticada investigación empírica con la reflexión sobre el comportamiento de un objeto que alterna su posición en función del tiempo.

Galileo efectuó varias mediciones de un cuerpo desplazándose por un plano inclinado y percibió aquello que, actualmente, contemplan todos los libros de física como si fuera algo extremadamente obvio, es decir, que el cuerpo se desplazaba a mayor velocidad a medida que el tiempo pasaba. Recurriendo a un plano inclinado para desacelerar el movimiento de caída, utilizó incesantemente toda su creatividad para obtener los datos de la posición de los objetos en función del tiempo durante el 
movimiento de descenso. Se constata entonces que la relación entre el espacio y el tiempo podría ser representada por una ecuación elemental, en la que la posición dependía del cuadrado del tiempo. La construcción de una teoría no se da sin más en el ámbito del pensamiento: es necesario investigar, medir, inferir, deducir y, por lo tanto, teorizar. El proceso para determinar una ley o registrar un evento se codifica en un lenguaje propio, no otro que el de las matemáticas, como podemos ver en Kittel, Knight, Ruderman (1965), Feynman (2005) e Hawking (1988).

Esto parece sugerir que la naturaleza posee un comportamiento propio e independiente en relación a quien la estudia: puede ser investigada, y cualquiera que lo haga debe llegar a los mismos resultados; como si la naturaleza pudiese ser descifrada por unos ojos atentos y bien adiestrados. Para ello se tornan imprescindibles la precisión y la eficiencia de los instrumentos que miden la longitud y los intervalos de tiempo. En efecto, Galileo necesitaba un instrumento de medición eficaz al modo de un hilo de agua que cae homogéneamente por un orificio u otro fenómeno regular. A diferencia de una regla, que puede ser fácilmente sometida a un patrón, las medidas de intervalos de tiempo deben basarse en algún evento que se repita constantemente de forma homogénea.

La observación de la oscilación regular de un péndulo abre la posibilidad de su uso con esta finalidad. Sin embargo, Galileo va más lejos cuando afirma en su obra Dos Nuevas Ciencias, que el péndulo posee la propiedad del isocronismo, esto es, la igual duración de la oscilación del péndulo no depende de su ángulo. Lo cierto es que después de Galileo se comprobó que ésta era una propiedad aproximada y no exacta, aunque ello nunca supusiera para él un problema metodológico insalvable. En consecuencia, habría de corresponder a sus predecesores afinar este tipo de aseveraciones. Con todo, esta forma de ver la naturaleza constituiría su gran legado. Galileo lanzó la idea del reloj con un sincronismo pendular, lo que estableció una base fundamental para cuantos físicos le sucedieron en la tarea de buscar instrumentos cada vez más exactos. De esa forma, la naturaleza podría ser investigada con la precisión que dichos instrumentos proporcionan.

La regularidad de los movimientos solar y lunar, que expresan un patrón de tiempo, es menor que la exactitud que se encuentra en un reloj: esto condujo al hombre a percibir las irregularidades de tales movimientos otrora observados con devoción. A partir de aquí, la naturaleza se puede investigar armado con una regla y un cronómetro: parece además responder a leyes mecánicas, con lo que cabe al hombre medir, analizar y teorizar, aunque no necesariamente en ese orden. En el método galileano, pensamiento y experiencia caminan juntos y esta circunstancia, según Elias, conlleva el nacimiento de "un nuevo concepto del tiempo, un tiempo físico, que se apartaba del antiguo concepto, relativamente mas unitario y centrado en el hombre" (Elias, 1988: 92).

Tras Galileo llega Newton, y el procedimiento para desvelar los secretos de la naturaleza persiste. Las regularidades aparentes se traducen en leyes y axiomas, que a su vez pueden generar nuevas conclusiones susceptibles de ser puestas a prueba. Es posible que la naturaleza obedezca a una rutina traducible en ecuaciones, es decir, codificable en un lenguaje físico-matemático. Con su obra maestra, 
Principios Matemáticos de Filosofía Natural, la Física adquiere una base sólida, y todo lo que habría de llegar en este campo siempre tendrá, desde ese momento, como referencia ineludible la imprescindible contribución newtoniana. Newton proporciona una justificación impecable para los argumentos de Galileo en relación al movimiento de los cuerpos y amplía la posibilidad de estudiar los astros, que también se transforman en objetos mecánicos para la Física. Nos ofrece una interpretación del tiempo. “...como elemento de orden eterno de la naturaleza que es dado a los hombres como cualquier otro objeto físico”, tal y como nos lo recuerda Elias (1988: 99). Para Newton el tiempo era inmutable, espacio y tiempo existían, y los hombres, los planetas y demás cuerpos celestes somos sus habitantes, dotados de un libre albedrío limitado por las leyes que regulan el cosmos como un gran reloj. En la presentación que se incluye en la segunda edición de los Principia, publicada en 1717, Newton afirma que "el tiempo absoluto, verdadero y matemático, por sí mismo, es de la naturaleza, fluye uniformemente sin relación con cualquier otra cosa externa y es también duración; el tiempo relativo, aparente y común es alguna medida de duración perceptible y externa (sea ella exacta y no uniforme) que se obtiene a través del movimiento y que es normalmente usado en lugar del tiempo verdadero, como una hora, un día, un mes o un año”. (Newton, 1990: 7)

El tiempo no volverá a ser más el mismo, los cielos no pertenecen a los dioses y los astros se transforman en objetos previsibles a través de un modelo mecánico. El retorno del cometa Halley es previsto por Edmund Halley, que descubre el periodo de su órbita usando las leyes de Newton y su modelo de fuerza gravitacional. Con estos cambios y la visión físico-matemática del universo, la naturaleza parece quedar bajo nuestro control; un universo mecánico, regido por leyes conocidas que nos permite producir maquinas más eficientes.

El reloj que nos proporcionó el desarrollo de las teorías sobre la Tierra y el Cosmos, aparentemente infinito, permite ahora una mayor eficiencia en la navegación. Para determinar la longitud de un barco en el mar es necesario comparar el tiempo local con el tiempo de Greenwich, considerado como referencia en estos casos. Se impone contar a bordo con un reloj exacto, pues un error de un minuto equivale aproximadamente a 28 kilómetros de imprecisión en la longitud. La eficiencia en la medida del tiempo es crucial para las navegaciones. Los relojeros pueden producir mejores relojes para que los navegadores puedan aventurarse en un mundo desconocido, para que los científicos puedan investigar y revelar los secretos de la naturaleza y para que los ingenieros puedan producir máquinas más ajustadas:

"Corroborando los estudios realizados por historiadores del tiempo físico, Elias detecta un cambio radical en la manera que la sociedad tiene de concebir el tiempo entre los siglos XVII y XVIII. Este cambio reside en la importancia de no señalar el tiempo sino medirlo. Aún con el perfeccionamiento de los relojes mecánicos a partir del siglo XIII, a mediados del siglo XIV un reloj exacto se atrasaba o adelantaba 15 minutos por día, a mediados del siglo XVI esa desviación se había reducido a 12 y en el siglo XVII a 8 minutos por día. A partir de 1660, con la utilización de los relojes de péndulo, la precisión pasó a ser no ya de minutos, sino de segundos. Se inauguraba así la época de la sincronización del tiempo por el uso de relojes a 
péndulo y cuerda, cada vez más exactos y que rápidamente pasaban de las torres de las iglesias a nuestras casas, nuestras carteras y posteriormente a nuestras pulseras”. (KULESZA, 2004: 5)

La Ciencia se aproxima al desarrollo tecnológico. Telescopios y microscopios son ahora más exactos y permiten observar estrellas lejanas y escudriñar los secretos fundamentales de la materia. Los sucesivos descubrimientos del mundo microscópico abren posibilidades aparentemente infinitas al uso tecnológico de estos conocimientos. Así, la ciencia que antes promovía reflexiones sobre el mundo -lo que es real y cómo puede ser conocido- comienza a ser dependiente de la tecnología.

El científico del siglo XX tiene que justificar su conocimiento y lo hace demostrando su aplicación a productos tecnológicos que producen ganancias económicas y sociales. Pocos se adentran en las cuestiones epistemológicas que atañen a su propio conocimiento. Si el trabajo produce riqueza además de máquinas más rentables y eficientes, el científico acabará por gozar del beneplácito de la comunidad. Las preocupaciones sobre la naturaleza del espacio y el tiempo, y la cuestión de la inserción del hombre en los mismos son relegadas a un segundo plano. Los astros dejaron de ser un patrón de tiempo aceptable y la cuestión fundamental que se plantea es cómo medir el tiempo de una manera más eficiente. El tiempo queda instrumentalizado: ahora es el tic-tac de los relojes. Para Elias (1998: 94), el avance de las ciencias físicas provocó que el tiempo "físico" se transformase en un paradigma del "tiempo" en general, pues este "tiempo físico" podría ser representado por cantidades aislables, factibles de ser medidas con rigurosa exactitud y figurar, junto a los resultados de otras mediciones, en cálculos matemáticos”.

A comienzos del siglo XX, surgió un nuevo concepto de tiempo en la Física. El tiempo absoluto newtoniano que fluye de manera independiente pierde pujanza dando paso a una teoría que lo concibe de manera geométrica. El espacio y el tiempo para la teoría de la relatividad son una expresión de la geometría de nuestro Universo y, en ese sentido, el tiempo se "especializa". Por su parte, la luz adquiere el rango de patrón temporal y espacial. Los conocimientos sobre la luz fundamentan los nuevos patrones de tiempo y espacio y al mismo tiempo dan lugar a una revolución en las comunicaciones. Toda la información se puede transmitir por luz a través de un cable de fibra óptica. Los patrones de tiempo y de espacio están ahora entrelazados por un valor constante: la velocidad de la luz en el vacío.

La Física también se rige por acuerdos y hechos históricos que se plantean a medida que se necesita mayor exactitud en las mediciones que se realizan. No hay nada en la Ciencia que nos permita afirmar, de manera categórica, que en el futuro no vayan a cambiar nuestros patrones o que no adoptemos nuevas visiones sobre el tiempo y el espacio. Aunque contemos con dos grandes teorías, micro y macro, sobre el cosmos que, de cierta manera, dieron paso a la revolución tecnológica del siglo XX, ambas no están acabadas y contienen lagunas que habrán de ser compensadas. Las bases de estas teorías, en las que subyace un concepto de tiempo 
geométrico mensurable mediante procesos que ocurren en lo más íntimo de la materia, proporcionan una visión del mundo enigmática y seductora y albergan un potencial tecnológico nunca antes visto.

El aparato tecnológico responsable de la medición del tiempo se apoya en una teoría compleja sobre el movimiento de la materia - ya sea la teoría cuántica, ya la relativa - y maneja una noción de tiempo distante de su historia y de los consensos que forman parte de su construcción como concepto. El tiempo, de acuerdo con Elias (1998: 97), adquiere vida propia al ejercer un poder coercitivo sobre el individuo: "Este se ve siempre obligado a regir su propio movimiento en el "tiempo", instituido por el grupo al que pertenece; grupo que cuanto más complejice y diferencie las cadenas de interdependencia funcional que unen a los hombres entre sí, dará paso a la dictadura de los relojes”.

\section{El concepto de tiempo para Norbert Elias}

La concepción del tiempo como un hecho trascendental, como una experiencia anterior y exterior, ligada - como en el principio newtoniano - a la idea de que la naturaleza está dotada de tiempo, o a estructuras a priori - como en la teoría kantiana - en la que el tiempo es solo subjetividad, es refutada por Elias. En su opinión, en ambos casos "el individuo se presenta solo frente al mundo, como un sujeto frente a un objeto que pretende conocer. Queda por saber si la naturaleza del sujeto o la del objeto desempeñan un papel decisivo en la construcción de las representaciones humanas, así como en la inserción de todos los hechos en el curso del tiempo" (Elias, 1998: 9-10).

También en el nivel del sentido común el tiempo es objetivado, al igual que sucede con otros datos de la experiencia colectiva como "sociedad", "cultura", lenguaje"; entidades éstas que parecieran existir de forma independiente a los seres humanos. A partir de datos empíricos y de diferentes fuentes, Elias construye una argumentación en la que defiende que a) el tiempo y el espacio no son facultades innatas en el ser humano y b) poseemos la capacidad para elaborar síntesis temporales progresivamente complejas. Para Elias el tiempo es algo socialmente construido por los propios instrumentos que lo miden, así como la experiencia humana en relación al tiempo se modifica continuamente. Para profundizar en esta comprensión del concepto tiempo, Elias sitúa como eje central de su exposición un interrogante fundamental: ¿cuál es la necesidad que tienen los hombres de determinar el tiempo y con qué finalidad? Al presentar posibles respuestas, Elias termina por proponer una categorización. Según el autor, la función principal de aquella necesidad sería la coordinación y la integración, primitivamente ejercida por los sacerdotes o los reyes, y que hoy se delega en los instrumentos de medición. Así pues, la inmutabilidad del tiempo deja de ser representada por una divinidad y se conecta con las leyes que representan el orden inmutable de la naturaleza.

Nuestras sociedades, en el desarrollo de su relación con el tiempo, presentan un alto grado de simbolización, institucionalización y síntesis. Simbolización en la 
medida en que no se pueden disociar las propiedades físicas de los instrumentos de medición del tiempo de su dimensión simbólica. Los relojes, en cuanto instrumentos de medida, encarnan el tiempo de la misma manera que las máscaras encarnan espíritus en las sociedades primitivas. Institucionalización en la medida en que la sincronización colectiva simbolizada en los calendarios discurre en paralelo a la formación de un Estado capaz de imponer esta uniformización. Y síntesis que permite evidenciar la relación de dos o más secuencias diferentes de transformación, de tal manera que una sirva de medida de tiempo para la otra u otras. Cuando se afirma que los relojes miden e indican de forma exacta el tiempo se hace evidente cómo un símbolo puede ser objetivado adquiriendo vida propia en el lenguaje y el pensamiento de los hombres a través de expresiones como "medir el tiempo" o "determinar el tiempo": así se realimenta el mito del tiempo como algo existente, determinable y computable por el hombre. A diferencia de otros seres, el hombre desarrolla lenguajes, esto es, elementos de representación del mundo a través del habla, la escritura y la imagen. El lenguaje alberga una dimensión representativa y posee la capacidad de producir palabras, textos o imágenes como expresiones creíbles del mundo ideal.

\section{Copérnico, Einstein y Elias}

El paso del tiempo en lo "físico" - una de las variables inmutables que los físicos miden y que desempeña un papel crucial en las ecuaciones matemáticas así como en las representaciones simbólicas de las "leyes de la naturaleza" - y en lo "social" instancia reguladora de los hechos sociales y modalidad de la experiencia humana generan la impresión que el primero de esos tiempos es "real” mientras el segundo no pasaría de una convención arbitraria. Para Elias, uno de los factores de aparente misterio del tiempo se encuentra en la persistencia de esta división: la distinción entre "natural" y "social" o "subjetivo" y "objetivo" es irrelevante y nada funcional. Echando mano de las diferentes consecuencias que trajeron consigo la revolución copernicana y la teoría de la relatividad en su aplicación al fenómeno de la existencia humana, Elias construye su crítica a la tradición filosófica de la explicación del tiempo; tradición centrada en la visión de un sujeto a-cósmico, cuya existencia parece independiente del universo físico.

Descartes y una extensa lista de filósofos convirtieron en absolutas y trascendentes, en a prioris sin fundamento empírico, representaciones históricamente construidas. En este sentido, Kant es blanco de duras críticas por su concepción del tiempo y el espacio como síntesis intelectual proveniente de la naturaleza humana y, por ello, innata. Según Elias, tanto Descartes como Kant se olvidaron del pasado y desecharon todo el conjunto de procesos de conocimiento que los había conducido hasta el estadio de síntesis en el que se ubicaban.

A contramano, Elias privilegia una concepción relacional en la que el tiempo y la realidad humana se estudian en constante interconexión prescindiendo de aquel enfoque en el que "naturaleza" y "realidad" aparecen disociadas. En sus propias 
palabras, una "revolución copernicana" que sustituye no solo el geocentrismo, sino también el egocentrismo ingenuo por una visión global de interrelaciones. Tal "revolución copernicana", abre la puerta a la "teoría de la relatividad", en la que las líneas de relación entre pasado, presente y futuro están siempre cambiando en la medida en que los propios sujetos de los hechos pasados, presentes y futuros se transforman o son remplazados por otros. Esta sería, según Elias, una concepción equivalente a la propuesta por Einstein, quien vio el tiempo como una forma de relación y no como una entidad independiente al hombre al uso de Newton.

De esta manera, si el tiempo constituye la cuarta dimensión, junto a las tres que definen el espacio, Elias sugiere que es necesario considerar la dimensión simbólica de la conciencia, es decir, una quinta dimensión que revela la comprensión humana del tiempo. La autorregulación "temporal" característica de las sociedades avanzadas no es un dato biológico ligado a la naturaleza, ni tampoco un dato metafísico, asociado a algún a priori imaginario. Es, ante todo, un dato social de la estructura de la personalidad, que, como tal, se hace parte integrante de la individualidad de cada uno de nosotros (Elias, 1998: 119).

\section{Conclusión}

La concepción del tiempo en la Física posee historicidad en el interior y en la especificidad del campo científico. Existe una evolución de este concepto, en diversas configuraciones, mas su naturaleza instrumental como resultado de las medidas que arroja el cronómetro parece inquebrantable. Desde un reloj impreciso, aunque capaz de proporcionar elaboradas teorías en su origen newtoniano, evolucionamos hasta el refinado reloj atómico. La luz emitida en este aparato por un átomo, en condiciones correctamente determinadas, produce un fenómeno regular generador de un patrón de medida de "intervalo de tiempo" que permite investigar la constitución de la materia tanto en el nivel del micro como del macrocosmos. Este reloj permite la medición de esta cuarta dimensión pudiendo, de esta forma, relacionarla con las tres dimensiones espaciales. Con esos datos, el físico investiga el comportamiento de los objetos en nuestro universo. Pese a que la quinta dimensión del pensamiento eliasiano no sea, aparentemente, contemplada por ninguna teoría física, ella subyace al propio pensamiento físico. A ojos de los no versados en estas materias, el lenguaje utilizado por los físicos resulta árido y no permite el diálogo con los símbolos matemáticos que intentan reproducir determinados fenómenos observables. Sin embargo, el mismo hombre que ambiciona descifrar los secretos del Cosmos posee historia - se encuentra, por lo tanto, situado en un universo simbólico - y siempre que observa el reloj con el propósito de conseguir una medida satisfactoria de intervalos temporales se encuentra con los símbolos de su tiempo. 


\section{Referencias Bibliográficas}

Elias, Norbert. Sobre o tempo. Rio de Janeiro: Jorge Zahar Ed., 1998

Feynman, Richard P., Leighton, Robert B., sands, Matthew. The Feynman Lectures on Physics. Addison-Wesley Publishing Company, 2005.

Kittel. Charles, Knight, Walter, Ruderman, Malvin A. Berkeley Physics Course, Mechanics. Vol. 1. Mcgraw-Hill College, 1965.

Kulesza, W. A.. A Quinta dimensão de Norbert Elias. Actas del VIII Simpósio

Internacional Processo Civilizador, História e Educação, Universidade Federal de Paraíba, 2004. Disponible en http://www.fef.unicamp.br/sipc/anais8/Wojciech\%20Andrzej\%20Kulesza\%20\%20UFPB.pdf. Última consulta: 21/09/2010.

Newton, Isaac. Principia: princípios matemáticos de filosofia natural. São Paulo: Nova Stella:EDUSP, 1990.

Stephen, Hawking. A Briefer History of Time. Bantam Books, 1988 